\title{
Perturbative approach to the Kondo effect in magnetic atoms on nonmagnetic substrates
}

\author{
Aaron Hurley, Nadjib Baadji, and Stefano Sanvito \\ School of Physics and CRANN, Trinity College, Dublin 2, Ireland
}

(Received 5 July 2011; published 22 September 2011)

\begin{abstract}
Recent experimental advances in scanning tunneling microscopy make the measurement of the conductance spectra of isolated and magnetically coupled atoms on nonmagnetic substrates possible. Notably, these spectra are characterized by a competition between the Kondo effect and spin-flip inelastic electron tunneling. In particular they include Kondo resonances and a logarithmic enhancement of the conductance at voltages corresponding to magnetic excitations, two features that cannot be captured by second-order perturbation theory in the electron-spin coupling. We have now derived a third-order analytic expression for the electron-spin self-energy, which can be readily used in combination with the nonequilibrium Green's function scheme for electron transport at finite bias. We demonstrate that our method is capable of semiquantitative description of the competition between Kondo resonances and spin-flip inelastic electron tunneling at a computational cost significantly lower than that of other approaches. The examples of $\mathrm{Co}$ and $\mathrm{Fe}$ on $\mathrm{CuN}$ are discussed in detail.
\end{abstract}

DOI: 10.1103/PhysRevB.84.115435 PACS number(s): 72.15.Qm, 71.10.-w, 73.40.Gk, 68.37.Ef

\section{INTRODUCTION}

The interaction between conduction electrons and localized spins in transition metals with partially filled $d$ shells is central to many low-temperature spin effects, which may underpin the development of spintronics and quantum information devices. When adsorbed on the surface of a metallic host, magnetic transition metal atoms exhibit various distinctive features in the conductance spectrum, which are indicative of many-body scattering between the conduction electrons and the localized spins. These manifest themselves as conductance steps at voltages corresponding to the quasiparticle energies of specific magnetic excitations and as zero-bias conductance peaks, known as Kondo resonances. The first are associated to spin-flip inelastic electron tunneling and can be described by second-order perturbation theory in the electron-spin coupling, ${ }^{1}$ but the second results from third-order effects due to the electron screening of the local spins.

Recent advances in scanning tunneling microscopy (STM) have enabled the detection of many-body scattering events in $\mathrm{Mn},{ }^{2} \mathrm{Fe},{ }^{3}$ and $\mathrm{Co}^{4,5}$ adatoms adsorbed on a $\mathrm{CuN}$ insulating substrate. The Co atom in particular shows interesting Kondo physics, and STM experiments have demonstrated the dependence of the Kondo peak on applied magnetic fields and also on the spatial extent of individual cobalt complexes. ${ }^{6}$ The reduced symmetry of the surface leads to significant magnetic anisotropy, especially for $\mathrm{Fe}$ and $\mathrm{Co}$. Fe is found to have a large easy-axis anisotropy [ $D<0$, see Eq. (2)], leading to a ground state spin close to that of the maximum $z$ component of the integer $S=2$. This results in four evenly spaced conductance steps in the spectrum. For Co, the large hard-axis anisotropy $(D>0)$ and the half-integer $S=3 / 2$ spin produce a doublet ground state. The measured zero-bias Kondo resonance is then due to spin transitions between the degenerate ground state levels. ${ }^{7}$

Theoretical attempts to reproduce these conductance spectra have focused largely on including second-order scattering events, which cannot account for Kondo resonances but fare well in reproducing the conductance steps and their relative intensities. ${ }^{1,8-13}$ Addressing Kondo physics in Co is more involved and one has to look for alternative techniques, such as Wilson's ${ }^{14}$ numerical renormalization group theory informed by density functional theory (DFT). This is an inherently nonperturbative approach which was developed to counteract the failings of perturbation theory at energies close to the Kondo temperature $T_{\mathrm{K}}$. The method creates a "logarithmically discretized" conduction band where energy intervals become exponentially smaller as the Fermi energy is approached. This makes the low-energy spectrum accessible and has formed the basis of many theoretical descriptions of the Kondo phenomenon in Co. ${ }^{15-17}$

Although these schemes recreate well the conductance spectra for magnetic atoms exhibiting the Kondo effect, they are numerically expensive. In this paper, we provide an alternative and inexpensive approach by going back to perturbation theory. Within our developed approach, we demonstrate a qualitative description of the behavior of the Kondo peaks for Co when this is exchange coupled to another magnetic atom. However, due to the aforementioned restrictions of the method we cannot claim an exact quantitative agreement with the experiments. In addition, for $\mathrm{Fe}$ we do find a quantitative description of the conductance step over-shooting subsequent to inelastic tunneling due to a magnetic excitation and to its decay as a function of bias. This was previously ascribed as originating from nonequilibrium effects, ${ }^{12}$ but here it is demonstrated to simply arise from the third-order contribution to the interacting self-energy.

The layout of this paper is as follows. First, we show how to extend the perturbative approach to the third order in the electron-spin scattering and derive an analytic expression for the scattering self-energy. This is then implemented within the nonequilibrium Green's function (NEGF) formalism ${ }^{18,19}$ for electron transport. Then we show in the results section how the derived expression performs when attempting to describe the STM conductance spectra of both Fe and Co on CuN. Finally we conclude. We have also included an appendix dedicated to detailed the derivation of the third-order expression for the interacting self-energy.

\section{MODEL}

The Hamiltonian describing the STM tip, a number of magnetic adatoms carrying localized spins $N_{A}$, and the 
nonmagnetic substrate can be divided into three components, namely a purely electronic part $H_{\mathrm{e}}$, a purely spin part $H_{\mathrm{sp}}$, and an electron-spin interaction part $H_{\mathrm{e}-\mathrm{sp}}$. These are given respectively by

$$
\begin{gathered}
H_{\mathrm{e}}=\sum_{k l \alpha} \varepsilon_{k l} a_{k l \alpha}^{\dagger} a_{k l \alpha}+\sum_{i \alpha}^{N_{A}} \varepsilon_{i} c_{i \alpha}^{\dagger} c_{i \alpha}+H_{\mathrm{tun}}, \\
H_{\mathrm{sp}}=\sum_{i}^{N_{A}}\left\{D_{i}\left(S_{i}^{z}\right)^{2}+E_{i}\left[\left(S_{i}^{x}\right)^{2}-\left(S_{i}^{y}\right)^{2}\right]\right\}, \\
H_{\mathrm{e}-\mathrm{sp}}=J_{\mathrm{sd}} \sum_{i}^{N_{A}} \sum_{\alpha, \alpha^{\prime}}\left(c_{i \alpha}^{\dagger}[\sigma]_{\alpha \alpha^{\prime}} c_{i \alpha^{\prime}}\right) \cdot \mathbf{S}_{i} .
\end{gathered}
$$

In $H_{\mathrm{e}}$, we assume that both the tip (t) and the substrate (s) are characterized by a single $s$-like band, so that the electronic structure (spin degenerate) is described by the creation (annihilation) operators $a_{k l \alpha}^{\dagger}\left(a_{k l \alpha}\right)$ and the energies $\varepsilon_{k l}(l=\{\mathrm{t}, \mathrm{s}\})$. Here, and later, $\alpha$ is the spin index for the conducting electrons $(\alpha=\uparrow, \downarrow)$. Note that the electronic structure of the tip and the substrate is spin degenerate. Similarly, the term $\sum_{i \alpha}^{N_{A}} \varepsilon_{i} c_{i \alpha}^{\dagger} c_{i \alpha}$ defines the electrostatic energy for the conducting electrons residing on the adatoms. Here $c_{i \alpha}^{\dagger}\left(c_{i \alpha}\right)$ creates (destroys) a conducting electron at the adatom site $i$ with spin $\alpha$, and $\varepsilon_{i}$ is the on-site energy, which is spin degenerate but can vary from site to site depending on the nature of the adatom.

The tunneling between the adatom and the tip (substrate) is accounted for by $H_{\text {tun }}$. This term describes the hopping from the tip (substrate) to one of the adatoms and essentially consists of a hopping integral $\gamma_{i}^{t-a}\left(\gamma_{i}^{s-a}\right)$. The leads can be replaced by an effective energy-dependent self-energy $\Sigma_{\mathrm{t}, \mathrm{s}}(E)$, which produces a constant broadening of the adatom density of states given by $\Gamma_{i}^{m}=\left(\gamma_{i}^{m}\right)^{2} / W$ (with $m=t-a$ or $s-a$, see Ref. 1). The broadening is thus constant around the Fermi energy $E_{\mathrm{F}}$, provided that the bandwidth $W$ is large compared to the coupling strength between the two leads and the adatom. Note that in general we always assume that both the tip and the substrate couple electronically to only one of the adatoms at a time, so that $\gamma_{i}^{m}=0$ except for $i=i_{0}$, for a given $i_{0}$. Note also that for most of the calculations shown here we consider a single adatom so that the index $i$ drops.

Finally, $H_{\mathrm{sp}}$ contains information on the magnetic anisotropy of the adatoms and includes both an axial $\left(D_{i}\right)$ and a transverse $\left(E_{i}\right)$ term. $H_{\mathrm{e}-\mathrm{sp}}$ couples the conducting electrons to the local spins $\mathbf{S}_{i}$ through an exchange interaction parameter $J_{\text {sd }}$ (assumed identical for the different adatoms). Note that the interaction is on-site, and it has a Heisenberg-like form. Note also that such a Hamiltonian (at least for $|S|=1 / 2$ ) can be derived from a two-band purely electronic one, in which the electronic structure of one of the bands includes a Hubbard term. The equivalence of the two models can be proved up to second order in $t / U$ by using the Wolff-Schrieffer transformation ${ }^{20}$ where $t$ and $U$ are the hopping and Coulomb repulsion integrals respectively.

As already discussed in Ref. 1, our strategy is that of incorporating all the inelastic contributions to the electronic structure in an interacting self-energy $\Sigma_{\text {int }}$. We then begin by considering a nonequilibrium system at finite temperature described at the level of the Keldysh formalism. ${ }^{21,22}$ The contour-ordered single particle many-body Green's function $G$ is defined as (note that for the sake of clarity we have dropped the site index $i$ )

$$
\left[G\left(\tau, \tau^{\prime}\right)\right]_{\sigma \sigma^{\prime}}=-i\left\langle\left|T_{C}\left\{c_{\sigma}(\tau) c_{\sigma^{\prime}}^{\dagger}\left(\tau^{\prime}\right)\right\}\right|\right\rangle,
$$

where |\rangle is the fully interacting ground state. This expression can be expanded up to the $n$th order ${ }^{27}$ in the electron-spin coupling as

$$
\begin{aligned}
& {\left[G\left(\tau, \tau^{\prime}\right)\right]_{\sigma \sigma^{\prime}}} \\
& =\sum_{n} \frac{(-i)^{n+1}}{n !} \int_{C} d \tau_{1} \ldots \int_{C} d \tau_{n} \\
& \quad \times \frac{\left\langle 0\left|T_{C}\left\{H_{\mathrm{e}-\mathrm{sp}}\left(\tau_{1}\right) \ldots H_{\mathrm{e}-\mathrm{sp}}\left(\tau_{n}\right) c_{\sigma}(\tau) c_{\sigma^{\prime}}^{\dagger}\left(\tau^{\prime}\right)\right\}\right| 0\right\rangle}{U(-\infty,-\infty)},
\end{aligned}
$$

where $U$ is the time-evolution unitary operator, and the time averages are now performed over the noninteracting ground state $|0\rangle$. We can now write explicitly the expansion up to the third order, which reads

$$
\begin{aligned}
{\left[G\left(\tau, \tau^{\prime}\right)\right]_{\sigma, \sigma^{\prime}}^{(3)}=} & \frac{(-i)^{4}}{3 !} J_{\text {sd }}^{3} \sum_{\alpha \alpha^{\prime}, \beta \beta^{\prime}, \gamma \gamma^{\prime}} \int_{C} d \tau_{1} \int_{C} d \tau_{2} \int_{C} d \tau_{3} \\
& \times\left\langleT _ { C } \left\{ c_{\sigma}(\tau) c_{\alpha}^{\dagger}\left(\tau_{1}\right) c_{\alpha^{\prime}}\left(\tau_{1}\right) c_{\beta}^{\dagger}\left(\tau_{2}\right) c_{\beta^{\prime}}\left(\tau_{2}\right) c_{\gamma}^{\dagger}\right.\right. \\
& \left.\left.\times\left(\tau_{3}\right) c_{\gamma^{\prime}}\left(\tau_{3}\right) c_{\sigma^{\prime}}^{\dagger}\left(\tau^{\prime}\right)\right\}\right\rangle \sum_{i, j, k}\left\langleT _ { C } \left\{ S^{i}\left(\tau_{1}\right) S^{j}\left(\tau_{2}\right)\right.\right. \\
& \left.\left.\times S^{k}\left(\tau_{3}\right)\right\}\right\rangle\left[\sigma^{i}\right]_{\alpha \alpha^{\prime}}\left[\sigma^{j}\right]_{\beta \beta^{\prime}}\left[\sigma^{k}\right]_{\gamma \gamma^{\prime}} .
\end{aligned}
$$

We then move our attention to the spin operators. Following the procedure presented in Ref. 1, these are decomposed into products of quasiparticle operators that obey the Fermi-Dirac statistics. The $i$ th component $(i=x, y, z)$ of the $\operatorname{spin} \mathbf{S}$ is written as

$$
S^{i}(\tau)=\sum_{m, m^{\prime}}\left\langle m\left|S^{i}\right| m^{\prime}\right\rangle d_{m}^{\dagger}(\tau) d_{m^{\prime}}(\tau),
$$

where $|n\rangle$ is an eigenstate of $H_{\mathrm{sp}}$, and $d_{n}^{\dagger}\left(d_{n}\right)$ creates (destroys) a quasiparticle in the same state. Thus the corresponding spin Green's function is of the form $\left[D\left(\tau, \tau^{\prime}\right)\right]_{m, m^{\prime}}=$ $-i\left\langle T_{C}\left\{d_{m}(\tau) d_{m^{\prime}}^{\dagger}\left(\tau^{\prime}\right)\right\}\right\rangle$. By substituting this expression back into Eq. (8) we obtain

$$
\begin{aligned}
{\left[G\left(\tau, \tau^{\prime}\right)\right]_{\sigma, \sigma^{\prime}}^{(3)}=} & \frac{(-i)^{4}}{3 !} J_{\mathrm{sd}}^{3} \sum_{\alpha \alpha^{\prime}, \beta \beta^{\prime}, \gamma \gamma^{\prime}} \int_{C} d \tau_{1} \int_{C} d \tau_{2} \int_{C} d \tau_{3}\left\langle T_{C}\left\{c_{\sigma}(\tau) c_{\alpha}^{\dagger}\left(\tau_{1}\right) c_{\alpha^{\prime}}\left(\tau_{1}\right) c_{\beta}^{\dagger}\left(\tau_{2}\right) c_{\beta^{\prime}}\left(\tau_{2}\right) c_{\gamma}^{\dagger}\left(\tau_{3}\right) c_{\gamma^{\prime}}\left(\tau_{3}\right) c_{\sigma^{\prime}}^{\dagger}\left(\tau^{\prime}\right)\right\}\right\rangle \sum_{m m^{\prime}, n n^{\prime}, l l^{\prime}} \\
& \times\left\langle T_{C}\left\{d_{m}^{\dagger}\left(\tau_{1}\right) d_{m^{\prime}}\left(\tau_{1}\right) d_{n}^{\dagger}\left(\tau_{2}\right) d_{n^{\prime}}\left(\tau_{2}\right) d_{l}^{\dagger}\left(\tau_{3}\right) d_{l^{\prime}}\left(\tau_{3}\right)\right\}\right\rangle \sum_{i, j, k}\left\langle m\left|S^{i}\right| m^{\prime}\right\rangle\left\langle n\left|S^{j}\right| n^{\prime}\right\rangle\left\langle l\left|S^{k}\right| l^{\prime}\right\rangle\left[\sigma^{i}\right]_{\alpha \alpha^{\prime}}\left[\sigma^{j}\right]_{\beta \beta^{\prime}}\left[\sigma^{k}\right]_{\gamma \gamma^{\prime}} \cdot
\end{aligned}
$$


We now perform the chronological contractions by using Wick's theorem for both the electron and spin bracket. In our previously published work, ${ }^{1}$ we have shown that in the electron brackets, the spin selection rules and the electronic spin degeneracy impose a vanishing contribution for any "fermion loop" contraction of the form $\left\langle c^{\dagger}(\tau) c(\tau)\right\rangle$. Therefore, we need only to consider the three Fock-like contributions to the electron bracket. These are all equal under exchange of contour indexes. The spin bracket has two Fock-like contributions, but in the following we restrict the derivation to only one of these for ease of description. Of course both the contributions will be included in the final result contained in Eq. (13). With this information in hand, the expression in Eq. (9) can be written in terms of the known noninteracting electron and spin Green's functions, $G_{0}$ and $D_{0}$ respectively,

$$
\begin{aligned}
{\left[G\left(\tau, \tau^{\prime}\right)\right]_{\sigma, \sigma^{\prime}}^{(3)}=} & i \frac{J_{\mathrm{sd}}^{3}}{2} \sum_{\beta, \gamma} \int_{C} d \tau_{1} \int_{C} d \tau_{2} \int_{C} d \tau_{3}\left[G_{0}\left(\tau, \tau_{1}\right)\right]_{\sigma \sigma}\left[G_{0}\left(\tau_{1}, \tau_{2}\right)\right]_{\beta \beta}\left[G_{0}\left(\tau_{2}, \tau_{3}\right)\right]_{\gamma \gamma}\left[G_{0}\left(\tau_{3}, \tau^{\prime}\right)\right]_{\sigma^{\prime} \sigma^{\prime}} \\
& \times \sum_{m, n, l}\left[D_{0}\left(\tau_{1}, \tau_{2}\right)\right]_{n, n}\left[D_{0}\left(\tau_{2}, \tau_{3}\right)\right]_{l, l}\left[D_{0}\left(\tau_{3}, \tau_{1}\right)\right]_{m, m} \sum_{i, j, k}\left\langle m\left|S^{i}\right| n\right\rangle\left\langle n\left|S^{j}\right| l\right\rangle\left\langle l\left|S^{k}\right| m\right\rangle\left[\sigma^{i}\right]_{\sigma \beta}\left[\sigma^{j}\right]_{\beta \gamma}\left[\sigma^{k}\right]_{\gamma \sigma^{\prime}}
\end{aligned}
$$

We can now use Dyson's equation to extract from Eq. (10) the third-order contribution to the interacting self-energy, which takes the form

$$
\begin{aligned}
\Sigma\left(\tau_{1}, \tau_{3}\right)^{(3)}= & 2 i J_{\text {sd }}^{3} \int_{C} d \tau_{2} G_{0}\left(\tau_{1}, \tau_{2}\right) G_{0}\left(\tau_{2}, \tau_{3}\right) \\
& \times \sum_{m, n, l} D_{n}\left(\tau_{1}, \tau_{2}\right) D_{l}\left(\tau_{2}, \tau_{3}\right) D_{m}\left(\tau_{3}, \tau_{1}\right) \\
& \times \sum_{i, j, k}\left(2 i \varepsilon_{i j k}\right)\left\langle m\left|S^{i}\right| n\right\rangle\left\langle n\left|S^{j}\right| l\right\rangle\left\langle l\left|S^{k}\right| m\right\rangle .
\end{aligned}
$$

Note that in order to simplify the notation, we have written the diagonal elements of the noninteracting spin self-energy as $D_{m}\left(\tau, \tau^{\prime}\right)$. In the same equation (12), we have also taken into account the electron spin degeneracy $\left(\left[G_{0}\right]_{\uparrow \uparrow}=\left[G_{0}\right]_{\downarrow \downarrow}\right)$ and traced over the spin indices. Finally, we note that $\operatorname{Tr}\left[\sigma^{i} \sigma^{j} \sigma^{k}\right]=2 i \varepsilon_{i j k}$.
The newly found Eq. (12) now needs to be expressed in terms of the real times $\left(t, t^{\prime}\right)$, so that a close expression for the energy resolved lesser and greater interacting self-energies can be explicitly written. Such a derivation is based on the Keldysh formalism for evaluation of time-contour integrals ${ }^{21,22}$ and is illustrated in the Appendix. Finally, in order to obtain the complete expression for the interacting greater and lesser self-energies, $\Sigma_{\text {int }}^{\lessgtr}$, one needs to include the second-order contributions

$$
\begin{aligned}
\Sigma_{\text {int }}^{\lessgtr}(E)^{(2)}= & -2 J_{\text {sd }}^{2} \sum_{m, n, i} P_{n}\left(1-P_{m}\right)\left|\left\langle m\left|S^{i}\right| n\right\rangle\right|^{2} \\
& \times G_{0}^{\lessgtr}\left(E \pm \Omega_{m n}\right),
\end{aligned}
$$

which have been previously derived in Ref. 1. Combining both second- and third-order contributions, we are left with the final total expression which reads

$$
\begin{aligned}
\Sigma_{\text {int }}^{\lessgtr}(E)= & -2 J_{\mathrm{sd}}^{2} \sum_{m, n, l} P_{l}\left(1-P_{m}\right) G_{0}^{\lessgtr}\left(E \pm \Omega_{m l}\right)\left\{\delta_{n l} \sum_{i}\left|\left\langle m\left|S^{i}\right| n\right\rangle\right|^{2}+2 i\left(\rho J_{\mathrm{sd}}\right) \sum_{i j k} \varepsilon_{i j k}\left\langle m\left|S^{i}\right| n\right\rangle\left\langle n\left|S^{j}\right| l\right\rangle\left\langle l\left|S^{k}\right| m\right\rangle\right. \\
& \left.\times\left[\ln \left|\frac{W}{\sqrt{\left(E+V \pm \Omega_{m n}\right)^{2}+\left(k_{\mathrm{B}} T\right)^{2}}}\right|+\ln \left|\frac{W}{\sqrt{\left(E+V \pm \Omega_{n l}\right)^{2}+\left(k_{\mathrm{B}} T\right)^{2}}}\right|\right]\right\},
\end{aligned}
$$

where $k_{\mathrm{B}}$ is the Boltzmann constant, $T$ is the temperature, $P_{l}$ is the population of the $|l\rangle$ spin state, and $V$ is the applied voltage. Note that the plus (minus) sign corresponds to < $(>)$. If we now assume that the adatom is much more strongly coupled to the substrate than to the STM tip, $\left(\gamma^{\mathrm{s}-\mathrm{a}} \gg \gamma^{\mathrm{t}-\mathrm{a}}\right)$ and $\varepsilon_{0} \gg E_{\mathrm{F}}$, we can approximate its density of states around $E_{\mathrm{F}}$ with a constant, $\rho=\left(\Gamma^{s-\mathrm{a}} / 2 \pi\right) /\left[\varepsilon_{0}^{2}+\left(\Gamma^{\mathrm{s}-\mathrm{a}}\right)^{2} / 4\right]$. The weak coupling to the STM tip also ensures that the spin system remains always close to equilibrium. ${ }^{12}$ This means that the adatom state always resides close to the ground state, i.e., that $P_{0} \sim 1$. The matrix elements $\left\langle m\left|S^{i}\right| n\right\rangle$, with $i=\{x, y, z\}$, determine the intensity of a given transition between an initial state $n$ and a final state $m$. We also note that the strength of the third-order interaction depends on the dimensionless parameter $\rho J_{\text {sd }}$ in agreement with the expression of Ref. 23 .

The calculation of the spin-inelastic conductance spectra of adatoms adsorbed on surfaces requires the implementation of the NEGF scheme including many-body scattering effects, which has been rigorously derived in Ref. 1 . In brief, the tunneling current at any terminal $i=\mathrm{t}, \mathrm{s}$ for the bias voltage $V$ can be calculated from

$$
\begin{gathered}
I_{i}=\int_{-\infty}^{+\infty} \bar{I}_{i}(E) d E, \\
\bar{I}_{i}(E)=\frac{e}{h} \operatorname{Tr}\left\{\left[\Sigma_{i}^{>}(E) G^{<}(E)\right]-\left[\Sigma_{i}^{<}(E) G^{>}(E)\right]\right\},
\end{gathered}
$$


where $\Sigma_{i}^{>}(E)=\left[1-f_{i}(E, V)\right] \Gamma_{i}$ and $\Sigma_{i}^{<}(E)=f_{i}(E, V) \Gamma_{i}$. Here $f_{i}(E, V)$ is the bias dependent Fermi function in each of the leads, $e$ is the electron charge, and $h$ is the Planck constant.

\section{RESULTS}

Having found a close formula for the interacting selfenergy, we can now proceed with the full NEGF formalism ${ }^{1}$ and calculate the conductance spectra of various adatoms on insulating substrates. In order to explore the performance of our perturbative approach in describing the Kondo physics contained in the third-order contribution to $\Sigma_{\text {int }}^{\lessgtr}$, we consider both $\mathrm{Co}$ and $\mathrm{Fe}$ adsorbed on $\mathrm{CuN}$. For these species, experimental data are available both for individual atoms and for interacting dimers. ${ }^{3-5}$

Figures 1 and 2 show the calculated conductance spectra respectively for single $\mathrm{Co}$ and single $\mathrm{Fe}$ atoms on $\mathrm{CuN}$ as the dimensionless parameter $\alpha=\rho J_{\text {sd }}$ is varied (note that the spectra are normalized relativel to the elastic-only conductance $G_{0}$ ). The $\alpha$ parameter is the coupling constant of the perturbation expansion as discussed extensively in our previous publication. ${ }^{1}$ This is varied by altering the coupling of the adatom to the substrate, i.e., by changing $\gamma^{\mathrm{s}-\mathrm{a}}$, which translates to modifying the value of the adatom density of states at the Fermi level.

Many of the other parameters of the model used in the present simulations have been extracted from the DFT calculations reported in Ref. 16. The broadening due to the coupling to the substrate (we neglect the one due to STM tip) is set initially at $\Gamma^{\mathrm{s}-\mathrm{a}}=0.1 \mathrm{eV}$ for both $\mathrm{Co}$ and Fe. In order to ensure consistency in our approximations $\left[\Gamma^{\mathrm{s}-\mathrm{a}}=\right.$ $\left.\left(\gamma^{\mathrm{s}-\mathrm{a}}\right)^{2} / W\right]$ we also set $\gamma^{\mathrm{s}-\mathrm{a}}=1.5 \mathrm{eV}$ and the substrate bandwidth to $W=20 \mathrm{eV}$. We then choose $\varepsilon_{0}=1 \mathrm{eV}$ to fulfill the criterion $\varepsilon_{0} \gg E_{\mathrm{F}}=0$. The magnitude of $J_{\mathrm{sd}}$ for both $\mathrm{Fe}$ and $\mathrm{Co}$ is held constant at $0.5 \mathrm{eV}$ (note that $J_{\mathrm{sd}}=$ $0.5 \mathrm{eV}$ is comparable to the value calculated from DFT for a Ni impurity sandwiched between Au leads ${ }^{24}$ ). Finally, the empirical parameters describing the axial $D$ and transverse $E$

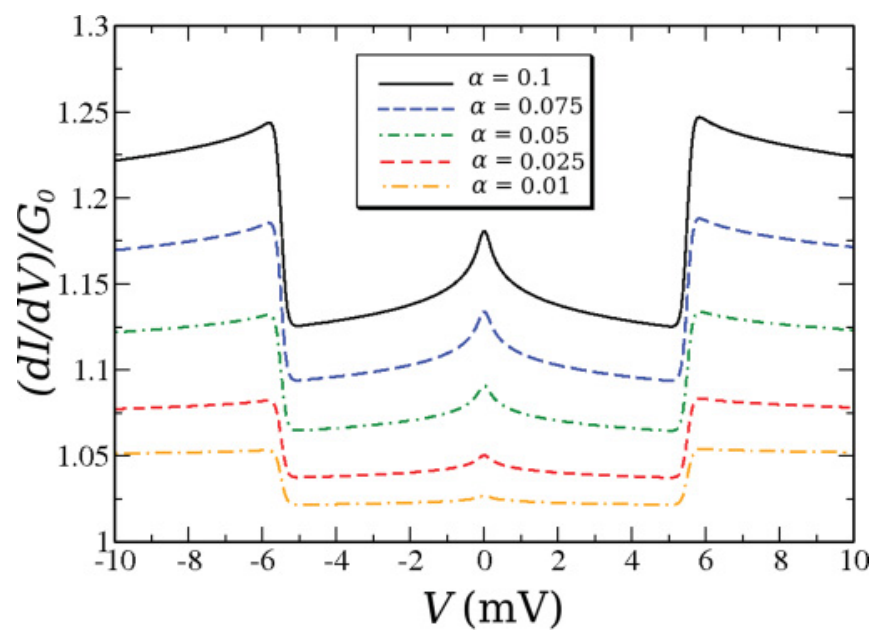

FIG. 1. (Color online) Normalized conductance spectrum for Co ( $S=3 / 2$ ) on $\mathrm{CuN}$ as $\alpha=\rho J_{\text {sd }}$ is increased. Note the emergence of a Kondo resonance at zero-bias, i.e., at the Fermi level. The curves are arbitrarily displaced for clarity.

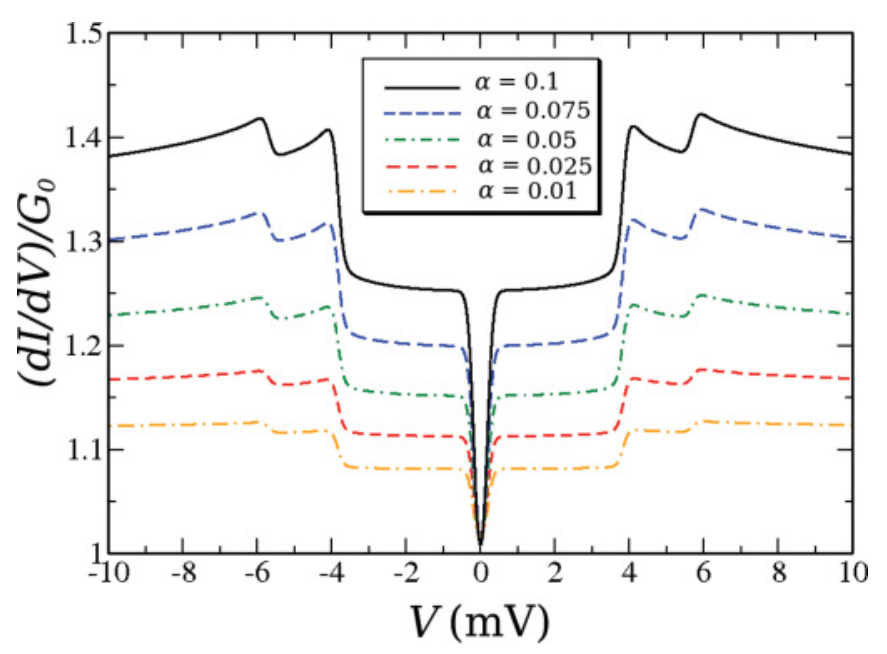

FIG. 2. (Color online) Normalized conductance spectrum for Fe ( $S=2)$ on $\mathrm{CuN}$ as $\alpha=\rho J_{\text {sd }}$ is increased. Note the transition from a constant conductance at the inelastic steps to a conductance that logarithmically decays after the excitation. The curves are arbitrarily displaced for clarity.

anisotropies are taken from the experimental fits of Refs. 3 and 4 and are $D_{\mathrm{Co}}=2.75 \mathrm{meV}, E_{\mathrm{Co}}=0 \mathrm{meV}, D_{\mathrm{Fe}}=$ $-1.53 \mathrm{meV}$, and $E_{\mathrm{Fe}}=0.31 \mathrm{meV}$, while the adsorbed atoms spins are $S_{\mathrm{Co}}=3 / 2$ and $S_{\mathrm{Fe}}=2$.

Let us start our discussion from the case of Co. The full diagonalization of the $H_{\mathrm{sp}}\left(S_{\mathrm{Co}}=3 / 2\right)$ Hamiltonian gives us a set of four $\left(2 S_{\mathrm{Co}}+1\right)$ eigenvalues and eigenvectors. In particular, the presence of a hard-axis anisotropy results in the following energy manifold $\varepsilon_{m}^{\text {Co }}=\{0.69,0.69,6.19,6.19\} \mathrm{meV}$, i.e., in a doubly degenerate ground state. It is then found that transitions between the degenerate ground states become allowed only on inclusion of the third-order term in Eq. (6). This is because of the selection rules imposed by the theory through the matrix elements $\left\langle m\left|S^{i}\right| n\right\rangle$. Such a transition appears in the spectrum of Fig. 1 in the form of a zero-bias Kondo peak, whose intensity increases as the value of $\alpha$ gets larger. The same feature is completely absent if one truncates the perturbation expansion to the second order. Note that the third-order contribution to the interacting self-energy scales as $\alpha^{2}$, while the second-order one goes as $\alpha$ (see also Ref. 1). Hence it follows that an increase of the adatom density of state (an increase of $\alpha$ ) promotes the third-order contribution to the self-energy. As such the enhancement of the Kondo peak intensity is directly related to the relative growth of the logarithmic divergence of $\Sigma_{\text {int }}^{\lessgtr}$ in Eq. (13). The same logarithmic divergence produces a second distinctive feature in the $d I / d V$ traces, namely the rise of the conductance following an inelastic excitation. This can be, for instance, seen in the conduction step at $6 \mathrm{meV}$. Such a step originates from the transition from the ground state to the first excited state. One may then note that first the conductance rises sharply at the voltage corresponding to the excitation energy and then slowly decays. Such a feature is absent if the perturbation expansion is truncated to the second order, ${ }^{1}$ and in that case the conductance step is essentially square (the conductance traces are similar to those found here for small $\alpha$ 's). Although we will come back on the agreement between our results and experiments, we note here that such 
conductance traces are qualitatively similar to those found in the STM experiments of Refs. 4 and 5, i.e., both the zero-bias Kondo peak and the increase in conductance at the inelastic transition voltages are observed.

The results for Fe are presented next in Fig. 2. This time $H_{\mathrm{sp}}\left(S_{\mathrm{Fe}}=2\right)$ has the five eigenvalues namely $\varepsilon_{m}^{\mathrm{Fe}}=\{-6.30$, $-6.12,-2.46,-0.60,0.18\} \mathrm{meV}$, so that the ground state is nondegenerate. At zero magnetic field, all the transitions allowed by the third-order expansion are resolved in the conductance traces. In this case there is no zero-bias Kondo peak as the ground state is nondegenerate. Near to the zero-bias region we now find a conductance dip, which corresponds to an inelastic transition between the first two lower lying spin states. Furthermore, as in the case of Co, here we also observe the presence of a logarithmic conductance increase at the inelastic steps in the $d I / d V$ traces (for instance, note the well pronounced one at $\sim 4 \mathrm{mV}$ ). Again, since such a feature is directly related to the third-order contribution to the interacting self-energy, it gets more pronounced as $\alpha$ is increased. The same feature is observed experimentally in all non-Kondo active adatoms, demonstrating the good level of description provided by the third-order expansion. Note that such a behavior was previously explained by invoking a nonequilibrium population of the Fe spin states. ${ }^{12}$ This explanation however conflicts with the fact that the same effect is seen also for low currents, ${ }^{3}$ namely when the adatom spin state resides close to the equilibrium. Therefore, we now re-interpret the experimental data as the manifestation of third-order Kondo corrections to the spin-flip inelastic tunneling spectra.

As a final test for our third-order self-energy we consider an exchanged coupled Co-Fe dimer, a situation already investigated experimentally in Ref. 5. In our formalism, this translates into including an additional Heisenberg-like term to $H_{\text {sp }}$, namely

$$
H_{\mathrm{H}}=J_{\mathrm{dd}}\left(\mathbf{S}_{\mathrm{Co}} \cdot \mathbf{S}_{\mathrm{Fe}}\right),
$$

where $J_{\mathrm{dd}}$ is the exchange coupling constant. Figures 3 and 4 show the conductance spectra calculated in the two situations where the tip is either positioned over Co (Fig. 3) or Fe (Fig. 4). We use a value for $\alpha=\rho J_{\text {sd }}=0.1$. In the figures, we also superimpose the experimental data ${ }^{5}$ for comparison.

Note that in general $J_{\mathrm{dd}}$ is small (not larger than $0.1 \mathrm{meV}$ ) so that any changes in the electronic levels of the combined $\mathrm{Co}-\mathrm{Fe}$ system are not resolved in the $\mathrm{mV}$ range, ${ }^{1}$ i.e., no new inelastic steps appear in the spectra. In general we notice a change in the $d I / d V$ traces of both $\mathrm{Co}$ and Fe as they are brought close together, i.e., as $J_{\mathrm{dd}}$ increases. For Fe, both the conductance steps around zero-bias and that at $0.18 \mathrm{mV}$ decrease in intensity with increasing $J_{\mathrm{dd}}$. In contrast, Fe itself acts as an effective magnetic field that splits the zero-bias Kondo resonance present in the spectrum of Co. Both these effects are observed in the experiments of Ref. 5. Notably Fe does not simply act as a source of magnetic field on Co, as seen in the inset of Fig. 3 for $J_{\mathrm{dd}}=0.1 \mathrm{meV}$. In the figure, one can clearly observe an additional Kondo peak emerging at zero bias in between the two principally split peaks. This is a unique feature of the exchange coupling between $\mathrm{Co}$ and $\mathrm{Fe}$. In fact, the exchange coupled Fe-Co dimer possesses $\left(2 S_{\mathrm{Co}}+1\right) \times\left(2 S_{\mathrm{Fe}}+1\right)=20$

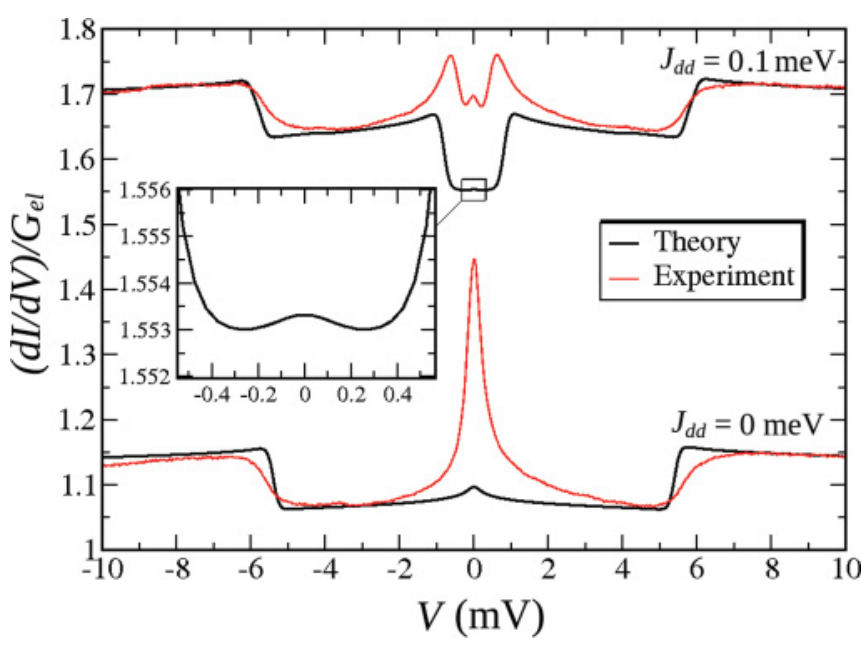

FIG. 3. (Color online) Normalized conductance spectrum for a Co adatom on $\mathrm{CuN}$ when it is exchange coupled to $\mathrm{Fe}$ as $J_{\mathrm{dd}}$ is increased $\left(J_{\mathrm{dd}}=0\right.$ means that there is no magnetic coupling between $\mathrm{Co}$ and $\mathrm{Fe}$ ). Note how the Co Kondo peak splits as the $\mathrm{Fe}$ atom acts as an effective magnetic field. The insert zooms in the zero-bias region. The calculated spectra are in black, while the corresponding experimental data from Ref. 5 are in red.

eigenvalues and additional allowed transitions appear at each of the atomic sites. For instance, for large $J_{\mathrm{dd}}$ the zero-bias region of the Co spectrum becomes completely dominated by a conductance dip. This originates from the opening of a spin transition between the ground state at $-5.686 \mathrm{meV}$ and the first excited state at $-5.379 \mathrm{meV}$. Such a transition, absent for the isolated Co adatom, has a spectral intensity much larger than that of the Kondo resonance, which therefore disappears from the spectrum.

We now wish to compare our data with the corresponding experimental spectra. ${ }^{5}$ Notably, whereas the calculated

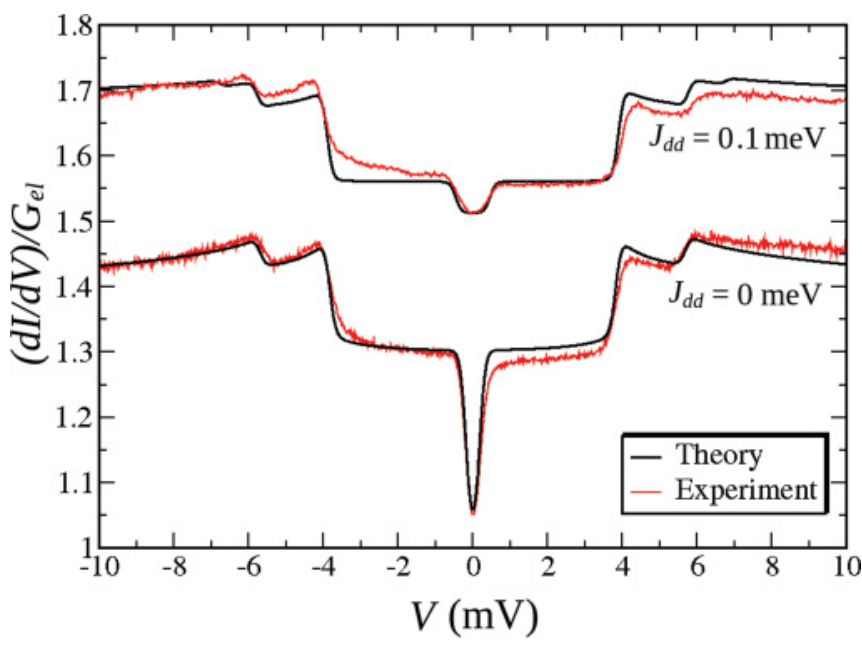

FIG. 4. (Color online) Normalized conductance spectrum for a $\mathrm{Fe}$ adatom on $\mathrm{CuN}$ when it is exchange coupled to Co as $J_{\mathrm{dd}}$ is increased $\left(J_{\mathrm{dd}}=0\right.$ means that there is no magnetic coupling between $\mathrm{Co}$ and $\mathrm{Fe}$ ). Note that the intensity of the conductance step at $0.18 \mathrm{mV}$ decreases with increased exchange coupling. The calculated spectra are in black, while the corresponding experimental data from Ref. 5 are in red. 
spectrum of $\mathrm{Fe}$ is in excellent quantitative agreement with the experimental one, the same cannot be said for that of Co, which only reproduces the experimental features at a qualitative level. In particular, the experimental Kondo resonance is much more pronounced than the calculated one (note that the parameter $\alpha$ has been set in order to reproduce the experimental step in the spectrum at $\sim 4 \mathrm{mV}$ ). At this point we can only speculate on the reasons for such a disagreement. Firstly, the $s-d$ model is valid only in the limit where the tunneling matrix element $t$ is small with respect to the adatom charging energy $U$. This is the case in which a Hubbard-like model can be mapped onto the $s-d$ one. ${ }^{20}$ Such a limit might not be satisfied for Co on CuN. In the event of a large $t / U$ ratio, a more rigorous two-body (Hubbard-like) approach needs to be employed to describe electrons in the localized $d$ states $^{25}$ or in the conducting $s$ states. $^{17}$

Notably, in the latter case of Misiorny et al., ${ }^{17}$ it was suggested that an increase in the exchange coupling parameter $J_{\text {sd }}$ may lead to a suppression of the Kondo peak at the Fermi level, which contrasts with what is found here. In our work, we chose to neglect any two-body terms in the conducting $s$-states as the inclusion of such would render the derivation of an analytic self-energy impossible. It has also been reported by Leuenberger et $a l .{ }^{26}$ and by Romeike et al. ${ }^{7}$ that the $s-d$ exchange coupling itself exhibits a large anisotropy such that $J_{\text {sd }}^{x, y} \ll J_{\text {sd }}^{z}$. This will certainly have an effect on the calculated conductance spectra, and as such will be investigated in a future work.

\section{CONCLUSIONS}

In conclusion, we have studied the effects of including thirdorder contributions in the interacting self-energy describing electron-spin coupling to the conductance spectra of transition metal atoms adsorbed on a $\mathrm{CuN}$ substrate. In particular, we have derived a close expression for the third-order electronspin self-energy within the NEGF formalism and a single band tight-binding model incorporating local Heisenberg exchange of conducting electrons to quantum spins. Two main features in the conductance spectra emerge from our formalism, namely a logarithmic decay of the conductance as a function of bias subsequent to a conductance step and zero-bias Kondo resonances. We obtain an almost perfect quantitative agreement for the spectrum of the non-Kondo active $\mathrm{Fe}$ adatom, but only a qualitative one for the Kondo active Co. The level of agreement is similar for both the situations where the adatoms are isolated or when they interact with each other via a Heisenberg-like exchange interaction.

The low computational effort needed by our method makes it a valuable alternative to full many-body treatments in describing spin inelastic phenomena at the atomic level. We also believe that our proposed scheme is amenable to be combined with first principles methods, i.e., it can form the basis for a fully quantitative theory of spin scattering in nanostructures. However, to improve the current results, we conclude that a more rigorous treatment, including two-body Hubbard terms in the Hamiltonian, might better describe the Co conductance spectra.
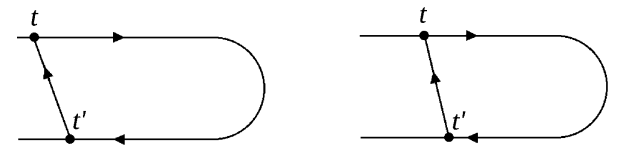

$G^{<}\left(t, t^{\prime}\right)$ and $G^{>}\left(t, t^{\prime}\right)$

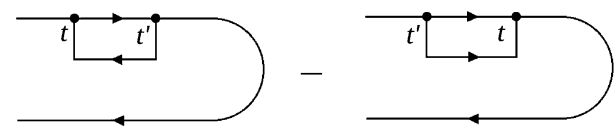

$$
G^{t}\left(t, t^{\prime}\right)
$$

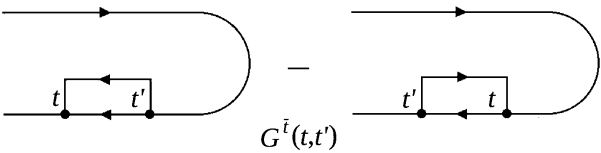

FIG. 5. Feynman rules for nonequilibrium Green's functions calculated over the contour running from $-\infty$ to $+\infty$. The four configurations are the lesser and greater Green's functions (a) and the time-ordered (b) and anti-ordered (c) Green's functions.

\section{ACKNOWLEDGMENTS}

This work is sponsored by the Irish Research Council for Science, Engineering \& Technology (IRCSET). N.B. and S.S. thank the Science Foundation of Ireland (Grant No. 08/ERA/I1759) and CRANN for financial support. Computational resources have been provided by the Trinity Centre for High Performance Computing (TCHPC). We wish to thank Alexander Otte and Cyrus Hirjibehedin for making the experimental data shown in Figs. 3 and 4 available to us.

\section{APPENDIX}

In this section, we will show how the integral in Eq. (12) is evaluated over the time-ordered contour $\tau_{2}$. The part of the self-energy in Eq. (12) which is of interest to us will be denoted by $\mathcal{I}$ and is written as follows:

$$
\begin{aligned}
\mathcal{I}\left(\tau_{1}, \tau_{3}\right)= & \sum_{m, n, l} D_{m}\left(\tau_{3}, \tau_{1}\right) \int_{C} d \tau_{2} G_{0}\left(\tau_{1}, \tau_{2}\right) \\
& \times G_{0}\left(\tau_{2}, \tau_{3}\right) D_{n}\left(\tau_{1}, \tau_{2}\right) D_{l}\left(\tau_{2}, \tau_{3}\right) .
\end{aligned}
$$

In order to express the quantity above in terms of real times we perform the integral over a contour going first from $-\infty \rightarrow+\infty$ and then from $+\infty \rightarrow-\infty^{21}$ so that only the ground state at $t=-\infty$ is well defined. The various branches along the contour result in four different Green's function configurations, which are illustrated in Fig. 5. These are respectively the lesser and the greater Green's functions $\left(G^{<}\right.$ and $G^{>}$) and both the time ordered and anti-ordered Green's functions $\left(G^{t}\right.$ and $\left.G^{\bar{t}}\right)$. The lesser $(<)$ contributions to $\mathcal{I}$ enter into Eq. (15) as

$$
\begin{aligned}
\mathcal{I}^{<}\left(t_{1}, t_{3}\right)= & \sum_{m, n, l} D_{m}^{>}\left(t_{3}, t_{1}\right)\left\{\int_{-\infty}^{+\infty} d t_{2} G_{0}^{t}\left(t_{1}, t_{2}\right) G_{0}^{<}\left(t_{2}, t_{3}\right)\right. \\
& \times D_{n}^{t}\left(t_{1}, t_{2}\right) D_{l}^{<}\left(t_{2}, t_{3}\right)+\int_{+\infty}^{-\infty} d t_{2} G_{0}^{\bar{t}}\left(t_{1}, t_{2}\right) G_{0}^{>}\left(t_{2}, t_{3}\right) \\
& \left.\times D_{n}^{\bar{t}}\left(t_{1}, t_{2}\right) D_{l}^{>}\left(t_{2}, t_{3}\right)\right\}
\end{aligned}
$$


where we define $G_{0}^{t}\left(t, t^{\prime}\right)=G_{0}^{<}\left(t, t^{\prime}\right)+G_{0}^{r}\left(t, t^{\prime}\right)$ and $G_{0}^{\bar{t}}\left(t, t^{\prime}\right)=G_{0}^{<}\left(t, t^{\prime}\right)-G_{0}^{a}\left(t, t^{\prime}\right)$. If we now insert these expressions into Eq. (A1), we obtain eight integrals that can be evaluated separately. These are

$$
\begin{aligned}
\mathcal{I}^{<}\left(t_{1}, t_{3}\right)= & \sum_{m, n, l} D_{m}^{>}\left(t_{3}, t_{1}\right)\left\{\int_{-\infty}^{+\infty} d \tau_{2} G_{0}^{r}\left(t_{1}, t_{2}\right) G_{0}^{<}\left(t_{2}, t_{3}\right) D_{n}^{r}\left(t_{1}, t_{2}\right) D_{l}^{<}\left(t_{2}, t_{3}\right)+\int_{-\infty}^{+\infty} d t_{2} G_{0}^{r}\left(t_{1}, t_{2}\right) G_{0}^{<}\left(t_{2}, t_{3}\right) D_{n}^{<}\left(t_{1}, t_{2}\right) D_{l}^{<}\left(t_{2}, t_{3}\right)\right. \\
& +\int_{-\infty}^{+\infty} d t_{2} G_{0}^{<}\left(t_{1}, t_{2}\right) G_{0}^{<}\left(t_{2}, t_{3}\right) D_{n}^{r}\left(t_{1}, t_{2}\right) D_{l}^{<}\left(t_{2}, t_{3}\right)+\int_{-\infty}^{+\infty} d t_{2} G_{0}^{<}\left(t_{1}, t_{2}\right) G_{0}^{<}\left(t_{2}, t_{3}\right) D_{n}^{<}\left(t_{1}, t_{2}\right) D_{l}^{<}\left(t_{2}, t_{3}\right) \\
& -\int_{-\infty}^{+\infty} d t_{2} G_{0}^{<}\left(t_{1}, t_{2}\right) G_{0}^{<}\left(t_{2}, t_{3}\right) D_{n}^{<}\left(t_{1}, t_{2}\right) D_{l}^{<}\left(t_{2}, t_{3}\right)+\int_{-\infty}^{+\infty} d t_{2} G_{0}^{<}\left(t_{1}, t_{2}\right) G_{0}^{<}\left(t_{2}, t_{3}\right) D_{n}^{<}\left(t_{1}, t_{2}\right) D_{l}^{a}\left(t_{2}, t_{3}\right) \\
& \left.+\int_{-\infty}^{+\infty} d t_{2} G_{0}^{<}\left(t_{1}, t_{2}\right) G_{0}^{a}\left(t_{2}, t_{3}\right) D_{n}^{<}\left(t_{1}, t_{2}\right) D_{l}^{<}\left(t_{2}, t_{3}\right)-\int_{-\infty}^{+\infty} d t_{2} G_{0}^{<}\left(t_{1}, t_{2}\right) G_{0}^{a}\left(t_{2}, t_{3}\right) D_{n}^{<}\left(t_{1}, t_{2}\right) D_{l}^{a}\left(t_{2}, t_{3}\right)\right\} .
\end{aligned}
$$

Importantly, several of these integrals cancel each other, while a few others can be grouped together by using the various definitions of $G \lessgtr$ and $G^{r / a} \cdot{ }^{27,28}$ By performing these simplifications, we finally obtain a much more compact expression

$$
\mathcal{I}^{<}\left(t_{1}, t_{3}\right)=2 \sum_{m, n, l} D_{m}^{>}\left(t_{3}, t_{1}\right)\left\{\int_{-\infty}^{+\infty} d t_{2} G_{0}^{r}\left(t_{1}, t_{2}\right) G_{0}^{<}\left(t_{2}, t_{3}\right) D_{n}^{<}\left(t_{1}, t_{2}\right) D_{l}^{<}\left(t_{2}, t_{3}\right)+\int_{-\infty}^{+\infty} d t_{2} G_{0}^{<}\left(t_{1}, t_{2}\right) G_{0}^{<}\left(t_{2}, t_{3}\right) D_{n}^{r}\left(t_{1}, t_{2}\right) D_{l}^{<}\left(t_{2}, t_{3}\right)\right\},
$$

which after performing the integration over $t_{2}$ becomes

$$
\begin{aligned}
\mathcal{I}^{<}\left(t_{1}, t_{3}\right)= & 2 \sum_{m, n, l} P_{l}\left(1-P_{m}\right) \iint d \omega d \omega^{\prime}\left\{G_{0}^{r}(\omega) G_{0}^{<}\left(\omega+\omega^{\prime}-\varepsilon_{l}\right) P_{n} \delta\left(\omega^{\prime}-\varepsilon_{n}\right)\right. \\
& \left.+G_{0}^{<}(\omega) G_{0}^{<}\left(\omega+\omega^{\prime}-\varepsilon_{l}\right) D_{n}^{r}\left(\omega^{\prime}\right)\right\} e^{i\left(\varepsilon_{m}-\omega-\omega^{\prime}\right)\left(t_{1}-t_{3}\right)} .
\end{aligned}
$$

Note here that, as we have previously shown, ${ }^{1} G_{0}^{r}(\omega)=1 /\left(\omega-\varepsilon_{0}+i \Gamma_{s}\right)$ and $G_{0}^{<}(\omega)=\Gamma_{s} f_{s}(\omega) /\left[\left(\omega-\varepsilon_{0}\right)^{2}+\Gamma_{s}^{2}\right]$. Again we assume that the coupling to the tip is negligible, leaving only the contribution to the broadening from the substrate. This is governed by the Fermi function $f_{s}(\omega)$. The spin subsystem is assumed to be weakly coupled to a surrounding heat bath kept at a temperature $T$, which ensures that the system remains in thermal equilibrium. Therefore, to a good approximation, we write $D_{m}^{r}(\omega)=1 /\left(\omega-\varepsilon_{m}+i k_{\mathrm{B}} T\right)$ and $D_{m}^{<}(\omega)=P_{n} \delta\left(\omega-\varepsilon_{m}\right)$. In the latter, we have assumed $k_{\mathrm{B}} T$ to be small enough that the lesser Green's function can be approximated by a delta function multiplied by the occupation of the specific spin state. With this information at hand, we note that the first term in the curly brackets in equation (A4) will not produce a logarithmic Kondo term but represents merely a small third-order correction to the scattering and can thus be neglected, as also found in Ref. 27. The second term contains the interesting third-order contribution, which after Fourier transform becomes

$$
\mathcal{I}^{<}(E)=2 \sum_{m, n, l} P_{l}\left(1-P_{m}\right) G_{0}^{<}\left(E+\varepsilon_{m}-\varepsilon_{l}\right) \int_{-W}^{+W} d \omega G_{0}^{<}(\omega) \operatorname{Re}\left[D_{n}^{r}\left(E-\omega+\varepsilon_{m}\right)\right] .
$$

Here we have only kept the real part of $D_{n}^{r}$, since we are only interested in the quantity $\operatorname{Im} \mathcal{I}^{r}(E)=\left[\mathcal{I}^{<}(E)+\mathcal{I}^{>}(E)\right] / 2$. The effect of $\operatorname{Re} \mathcal{I}^{r}(E)$ on the conductance is negligible in the case of $\varepsilon_{0} \gg E_{\mathrm{F}} .{ }^{1}$ It is also due to this fact that the density of states $\rho$ around $E_{\mathrm{F}}$ is approximately constant and therefore can be taken outside of the integral in Eq. (A7). This leads to a new expression for $\mathcal{I}^{<}(E)$, which reads

$$
\mathcal{I}^{<}(E)=2 \sum_{m, n, l} P_{l}\left(1-P_{m}\right) G_{0}^{<}\left(E+\varepsilon_{m}-\varepsilon_{l}\right) \rho \int_{-W}^{-V} d \omega \operatorname{Re}\left[\frac{1}{\left(E-\omega+\varepsilon_{m}-\varepsilon_{n}\right)+i k_{B} T}\right] .
$$

Finally, we perform the integral and also repeat the entire procedure for $\mathcal{I}^{>}(E)$ and arrive at the final expression, which reveals the logarithmic divergences present at the allowed transitions

$$
\mathcal{I}^{\lessgtr}(E)=-2 \sum_{m, n, l} P_{l}\left(1-P_{m}\right) G_{0}^{\lessgtr}\left(E \pm \Omega_{m l}\right) \rho \ln \left[\frac{W^{2}}{\left(E+V \pm \Omega_{m n}\right)^{2}+\left(k_{B} T\right)^{2}}\right] .
$$

\footnotetext{
${ }^{1}$ A. Hurley, N. Baadji, and S. Sanvito, Phys. Rev. B 84, 035427 (2011).

${ }^{2}$ C. F. Hirjibehedin, C. P. Lutz, and A. J. Heinrich, Science 312, 1021

${ }^{3}$ C. F. Hirjibehedin et al., Science 317, 1199 (2007).

${ }^{4}$ A. F. Otte et al., Nature Phys. 4, 847 (2008). (2006).

${ }^{5}$ A. F. Otte, M. Ternes, S. Loth, C. P. Lutz, C. F. Hirjibehedin, and A. J. Heinrich, Phys. Rev. Lett. 103, 107203 (2009).
} 
${ }^{6}$ J. J. Parks et al., Science 328, 1370 (2010).

${ }^{7}$ C. Romeike, M. R. Wegewijs, W. Hofstetter, and H. Schoeller, Phys. Rev. Lett. 96, 196601 (2006)

${ }^{8}$ J. Fernandez-Rossier, Phys. Rev. Lett. 102, 256802 (2009).

${ }^{9}$ J. Fransson, O. Eriksson, and A. V. Balatsky, Phys. Rev. B 81, 115454 (2010).

${ }^{10}$ N. Lorente and J. P. Gauyacq, Phys. Rev. Lett. 103, 176601 (2009).

${ }^{11}$ F. Delgado, J. J. Palacios, and J. Fernandez-Rossier, Phys. Rev. Lett. 104, 026601 (2010).

${ }^{12}$ B. Sothmann and J. Konig, New J. Phys. 12, 083028 (2010).

${ }^{13}$ M. Persson, Phys. Rev. Lett. 103, 050801 (2009).

${ }^{14}$ K. G. Wilson, Rev. Mod. Phys. 47, 773 (1975).

${ }^{15}$ R. Zitko, R. Peters, and T. Pruschke, New J. Phys. 11, 053003 (2009).

${ }^{16}$ R. Zitko and T. Pruschke, New J. Phys. 12, 063040 (2010).

${ }^{17}$ M. Misiorny, I. Weymann, and J. Barnas, Phys. Rev. Lett. 106, 126602 (2011).

${ }^{18}$ S. Datta, Quantum Transport: Atom to Transistor (Cambridge University Press, Cambridge, 2005).
${ }^{19}$ A. R. Rocha, V. M. García-Suárez, S. Bailey, C. Lambert, J. Ferrer, and S. Sanvito, Phys. Rev. B 73, 085414 (2006).

${ }^{20}$ J. R. Schrieffer and P. A. Wolff, Phys. Rev. 149, 491 (1966).

${ }^{21}$ L. V. Keldysh, Sov. Phys. JETP 20, 1307 (1965).

${ }^{22} \mathrm{H}$. Haug and A. P. Jauho, Quantum Kinetics in Transport and Optics of Semiconductors (Springer, New York, 1996).

${ }^{23}$ F. Elste and C. Timm, Phys. Rev. B 81, 024421 (2010).

${ }^{24}$ P. Lucignano, R. Mazzarello, A. Smogunov, M. Fabrizio, and E. Tosatti, Nat. Mater. 8, 563 (2009).

${ }^{25}$ O. Újsághy, J. Kroha, L. Szunyogh, and A. Zawadowski, Phys. Rev. Lett. 85, 2557 (2000).

${ }^{26}$ M. N. Leuenberger and E. R. Mucciolo, Phys. Rev. Lett. 97, 126601 (2006).

${ }^{27}$ G. D. Mahan, Many-Particle Physics, 2nd Ed. (Plenum Press, New York, 1990).

${ }^{28}$ T. Frederiksen, M. Brandbyge, N. Lorente, and A. P. Jauho, J. Comput. Electron. 3, 423 (2004). 